Fecha de recepción: octubre 2008 Fecha de aceptación: noviembre 2008 Versión final: diciembre 2008

\section{El beneficio público de las relaciones públicas. Un escenario en el que todos ganan}

\author{
Elisabeth Lewis Jones ${ }^{(*)}$
}

\footnotetext{
${ }^{(*)}$ Elisabeth Lewis-Jones es la Presidenta del Chartered Institute of Public Relations (CIPR / Instituto de Relaciones Públicas de Gran Bretaña) y Directora de Liquid Public Relations. El Instituto de Relaciones Públicas de Gran Bretaña, fundado en 1948, es la asociación profesional líder de la industria de las Relaciones Públicas del Reino Unido. El CIPR es, asimismo, la asociación más grande de su clase en Europa.
}

Resumen: A continuación se reproduce el contenido traducido al español, de la exposición realizada por Elisabeth Lewis Jones en la 'Primera Conferencia Internacional de Relaciones Públicas', organizada en ocasión de la celebración del $50^{\circ}$ aniversario por el Consejo Profesional de Relaciones Públicas de la República Argentina, en Buenos Aires, el 26 de agosto de 2008, con la participación de referentes internacionales de la industria. Lewis Jones, en su función de Presidenta del Chartered Institute of Public Relations (CIPR / Instituto de Relaciones Públicas de Gran Bretaña) reflexiona acerca del escenario global e internacional de las relaciones públicas, destacando el aporte que éstas realizan al desarrollo social a través de la gestión de una comunicación efectiva.

Palabras claves: Chartered Institute of Public Relations - Consejo Profesional de Relaciones Públicas de la República Argentina - Relaciones Públicas - Reputación Corporativa - Relaciones con los Inversores - Comunicación Interna - Comunicación de Marketing - imagen corporativa - organización - gestión comunicacional - planes de comunicación - públicos - comunicación corporativa - comunicación empresaria - comunicación institucional

[Resúmenes en inglés y portugués en la página 130]

A continuación, la exposición de Elisabeth Lewis Jones, que se reproduce con la autorización del Chartered Institute of Public Relations Relations de Gran Bretaña y del Consejo Profesional de Relaciones Públicas de la República Argentina:

Es probablemente más realista que pesimista, decir que la perspectiva económica para los próximos doce meses es incierta.

Quizás, en forma irónica, es en tiempos como estos, en los que las empresas buscan resguardarse de los vaivenes económicos y contemplan implementar recortes en ciertas áreas, cuando sus inversiones en Relaciones Públicas deberían incrementarse.

Muchas organizaciones -ya sean grandes o pequeñas, públicas o privadas- reconocen ahora la importancia del manejo de su reputación a través de una efectiva comunicación, que es, como es bien sabido, el campo de acción propio de las Relaciones Públicas. 
Que sus clientes, proveedores y empleados elijan comprar su producto o servicio, o asociarse con usted, depende de cómo ellos perciben a su organización, y lo que probablemente es más importante, de cómo ellos ubican a su compañía en relación a sus competidores.

Y a medida que el mundo se hace más y más pequeño y que los productos y servicios compiten a nivel global, los límites entre los países disminuye y la necesidad de una integración global de las Relaciones Públicas se incrementa; más que nunca en el complejo clima económico actual.

Para los profesionales de las Relaciones Públicas, el viejo enunciado "piense globalmente a nivel local" ha crecido en importancia. Debemos tener una visión global, y observar el macro escenario cuando planeamos nuestras estrategias y al mismo tiempo llegar hasta los niveles más básicos cuando trabajamos con mensajes a nivel local.

En junio de 2008, en Londres, el Chartered Institute of Public Relations organizó la 5 Conferencia y Festival Internacional de Relaciones Públicas en torno a la temática "la ventaja pública de las Relaciones Públicas: el desarrollo social a través de la comunicación efectiva".

Entre sus principales conclusiones, la conferencia destacó que como industria estamos definitivamente mucho más atentos a las cuestiones culturales. Tomamos en cuenta los aspectos sensibles como la raza, el lenguaje y la conducta individual de los mercados locales a pesar de que debamos promocionar marcas internacionales.

Sin embargo, no todo sucede todavía en forma tan sencilla. En los niveles más elementales, los comunicadores aún cometen errores, malinterpretando a los mercados locales y sus condiciones culturales. El reciente caso europeo de un flamante CEO alemán quien anunció que su ofensiva en el mercado de las Relaciones con Inversores de la ciudad de Londres sería denominado "Operación Blitz" -una clara referencia a la Segunda Guerra Mundial- es un ejemplo de este tipo de error. Lo que podría haber sido un toque de humor, se convirtió en una pérdida de respeto hacia su persona y le significó la pérdida de su trabajo.

La pasión por lo local puede ser peligrosa y generar divisiones; pero su aplicación adecuada ha producido un renacimiento de las culturas minoritarias con extraordinarias y ampliamente pacíficas historias exitosas. No se puede, por ejemplo, comunicar en Europa basando los planes y estrategias en Frankfurt, Londres o Bruselas sin tener en cuenta las diversas culturas europeas y sus formas de comunicarse.

La importancia de entender las diferencias culturales en relación a la comunicación es la razón por la cual el Chartered Institute of Public Relations en Gran Bretaña trabaja de forma tan cercana con el GA -Global Alliance for Public Relations and Communication Management-, que cuenta con más de 60 asociaciones nacionales de Relaciones Públicas entre sus miembros. A través de la GA estas asociaciones nacionales pueden aprender acerca de las diferencias culturales, su impacto en el negocio de las comunicaciones y en el desarrollo de la industria.

La GA no reemplaza a las asociaciones nacionales ni duplica su trabajo. A través de la Alianza, las asociaciones nacionales pueden compartir su trabajo con otras asociaciones nacionales y con sus miembros a nivel individual en forma igualitaria y equitativa.

La GA asume su obligación de brindar apoyo a sus miembros individuales, a las Relaciones Públicas como profesión y a la comunidad en general. La institución considera valioso el rol que desempeña en el mantenimiento de altos estándares de calidad profesional; facilitando el entendimiento cultural y apoyando una industria que opera a favor del interés y el beneficio público.

Y es por este motivo, que la Conferencia y Festival Internacional de Relaciones Públicas se focalizó en la problemática del beneficio público que constituye al mismo tiempo, una de las temáticas que toca más de cerca a la CIPR en Gran Bretaña. 
En primer lugar, las Relaciones Públicas se han convertido en la voz de quienes normalmente no tienen un perfil comunicador. Las PR brindan a grupos de influencia, pequeñas organizaciones y ONG herramientas que les permiten comunicarse con sus públicos, con las grandes corporaciones y con el sector público.

En segundo lugar, en Gran Bretaña, el Instituto posee el aval de la Corona a través del capítulo real que nos fue otorgado por su Majestad la reina de Inglaterra. Este reconocimiento nos fue otorgado por nuestra representatividad en la industria de las Relaciones Públicas avalada por nuestros 10.000 miembros activos; lo que nos permite dar cuenta de nuestra diversidad y nuestra oferta de liderazgo a través de la capacitación, certificaciones, perfiles y desarrollo de técnicas y políticas. Hemos demostrado que hemos elevado los estándares profesionales y que hemos logrado que el rol de las Relaciones Públicas en los negocios y la sociedad del Reino Unido sea mejor comprendido.

Justamente la semana pasada, recibimos la notificación que la Cámara de los Lores manifestó su acuerdo en relación a las actividades de nuestra organización, ubicándonos en el mismo nivel de otros cuerpos profesionales.

En muchos aspectos, nuestra organización es muy similar al Consejo Profesional de Relaciones Públicas de la República Argentina. Nosotros hemos celebrado nuestro $60^{\circ}$ aniversario -al mismo tiempo que ustedes celebraron sus 50 años de actividad-. En el Reino Unido, la industria se ha ido profesionalizando en forma creciente y actualmente sus integrantes son graduados altamente calificados. El CIPR se ha desarrollado logrando un amplio reconocimiento, quizás en forma más visible en la última década. Durante este tiempo, hemos tenido un significativo crecimiento, duplicando nuestros asociados a 10.000 miembros que trabajan en diferentes sectores de la industria; hemos certificado a un creciente número de profesionales de las Relaciones Públicas; hemos desarrollado y expandido nuestros cursos de capacitación y calificaciones profesionales a nivel nacional e internacional. Actualmente, contamos con seis centros internacionales en Bulgaria, Chipre, Egipto, Eslovenia, Nigeria y Ucrania. Asimismo, el CIPR Diploma -nuestro curso de calificación profesional- puede realizarse online facilitando a personas de todo el mundo la obtención de una calificación CIPR.

Pero el Capítulo Real no es concedido con la intención de beneficiar a la organización que la obtiene -más allá de que en nuestro caso nos haya beneficiado- sino que tiene como objetivo beneficiar a la sociedad en su conjunto.

Como institución -tal como figura en nuestra acta constitutiva- promovemos activamente el conocimiento público acerca de la contribución efectiva de las Relaciones Públicas en alentar las comunicaciones éticas potenciando la eficacia y rendimiento de todos los sectores de la economía.

En síntesis, por decreto Real representamos “al beneficio público de las Relaciones Públicas". Tenemos el deber de salir y predicar acerca de los beneficios de las Relaciones Públicas a todos nuestros públicos.

Como ustedes, para poner en evidencia el beneficio público de las Relaciones Públicas, nosotros debemos demostrar que representamos a la industria. La representatividad ha sido siempre uno de los temas más difíciles para las asociaciones de Relaciones Públicas. Por un lado, existen muchos profesionales trabajando por ejemplo en Relaciones con los Inversores o Comunicaciones Internas o incluso en Marketing quienes claramente hacen lo que nosotros consideramos "Relaciones Públicas", pero que no quieren ser identificados como relacionistas públicos, especialmente desde que el concepto de relaciones públicas ha estado sufriendo un quiebre en los medios británicos en los últimos años; lo cual afecta la moral de las personas y su sentido de identidad.

Existen también otras personas, que si bien no son miembros de ninguna asociación profesional se identifican con el trabajo de un instituto como el nuestro o una asociación como la vuestra. Debo 
confesar que esta gente me irrita. Algunos de ellos son operadores independientes; otros sin embargo, están en una posición influyente y se han convertido en modelos a seguir para los jóvenes. Estas personas deberían saber más, deberían reconocer el rol clave que tienen las asociaciones profesionales en el desarrollo de nuestra profesión y por lo tanto en sus negocios. Y ellos precisamente son, con frecuencia, los primeros en lamentarse de la falta de reconocimiento que tiene la profesión en todos sus aspectos.

En el otro extremo del espectro, están aquellos que son básicamente publicistas o modelos o quienes trabajan en agencias de gacetillas y que se autodenominan "relacionistas públicos", y con quienes no nos interesa mantener ninguna relación salvo educarlos para mejorar sus conocimientos sobre la profesión.

Nosotros podemos sin embargo afirmar que somos representativos del negocio en otras formas. La edad promedio de los miembros del CIPR es de 35 años, en tanto que en la industria en su conjunto el promedio es de 40 años. El $62 \%$ de nuestros miembros son mujeres, de acuerdo a las investigaciones realizadas recientemente.

Es importante también que las asociaciones de Relaciones Públicas demuestren su liderazgo, especialmente en la búsqueda del bien público. Esto significa estar involucrados en informar, interpretar y asesorar acerca del desarrollo de políticas en aquellas áreas en las cuales los profesionales de las relaciones públicas poseen un legítimo interés. A través de este asesoramiento, leyes que se originaron principalmente en Bruselas o Londres -como las de libertad de expresión; medios de comunicación, regulación de derechos de autor o leyes diseñadas para mejorar la comunicación con los empleadosestán ahora disponibles libremente y sin vinculaciones partidarias o políticas.

Un área que estamos impulsando vigorosamente como parte de nuestra representatividad es la diversidad.

Hemos lanzado un programa de diversidad con el objetivo de agregar valor a las numerosas iniciativas existentes en este campo, en parte, porque estamos profundamente preocupados por la aparente falta de oportunidades de desarrollo de carrera en las comunidades de países emergentes. Pero también, porque reconocemos que la práctica de las Relaciones Públicas está perdiendo una real oportunidad de negocios mientras no comprenda las necesidades de comunicación de estas comunidades. Las Relaciones Públicas juegan un rol único en fomentar ese aspecto de nuestra democracia.

Estas y otras iniciativas emanadas de las distintas asociaciones muestran la importancia de las Relaciones Públicas para nuestra economía y nuestra sociedad. Aún no hemos logrado recalcar este mensaje clave con la suficiente fuerza. De hecho, en Gran Bretaña y en Europa en general todavía no ha sido lo suficientemente comprendido.

$\mathrm{Y}$ en la medida en que enviamos este mensaje a una audiencia mayor, por el bien público, debemos asegurarnos que nuestra propuesta es sólida y cumple con las expectativas. De hecho, estamos produciendo actualmente más de 300 graduados en relaciones públicas al año y tenemos un esquema de desarrollo profesional continuo.

La sociedad británica en su conjunto, está hoy más focalizada en el concepto de "desarrollo Profesional continuo" y "educación permanente", que en los exámenes puntuales y las acreditaciones. Nosotros tenemos usualmente 700 miembros registrados en un proceso formal de CPD y todos nuestros métodos están instrumentados con el objetivo de maximizar la adopción e incorporación del proceso por parte de los involucrados.

Y aún así... a pesar de toda esta actividad, las Relaciones Públicas son aún caratuladas como banales y complementarias, o como una forma de disfrazar la verdad. 
En parte es nuestro error. En amplios sectores de la comunidad de las Relaciones Públicas existe una gran resistencia a la idea de las calificaciones y aún de la capacitación. Nuestra encuesta realizada en 2003 mostró que el 50\% de los consultados no realiza aparentemente provisiones presupuestarias para la capacitación de su staff. Otros continúan siendo reticentes a las carreras de Relaciones Públicas por ejemplo. El problema es menos agudo a nivel interno, especialmente en el sector público.

Siguiendo un proverbio inglés, esto puede ser un síndrome de "lo mejor como enemigo de lo bueno". Algunos de nuestros más destacados líderes de las relaciones públicas pertenecen a una generación que dio sus primeros pasos mucho antes de la creación de programas educativos de relaciones públicas; y otros más jóvenes son personas que se destacan como generalistas que podrían haber sido exitosos en cualquier profesión liberal.

Hacer realidad este cambio es nuestra estrategia a largo plazo. En un escenario donde la prensa es indiferente y hostil al mismo tiempo y donde las comunidades de negocios y política se encuentran en general mal informadas, este cambio no puede suceder de un día para otro. Pero con la calidad de las personas que van incorporándose a la profesión y con la intensa focalización en la reputación, la batalla puede ser ganada, aunque en forma muy lenta.

Además, creo apasionadamente en el poder de las Relaciones Públicas, en su poder para hacer el bien, para hacer todas aquellas cosas que sostenemos en nuestra carta constitutiva con la cual estamos comprometidos, y en su poder para lograr el bien público.

Y creo apasionadamente que sólo trabajando en equipo y en un mutuo reconocimiento en asociaciones como las nuestras, y en la Global Alliance y el CIPR, podremos alcanzar cualquiera de esos objetivos.

Soy una apasionada de la industria de las PR en el Reino Unido, de modo que pido disculpas por la digresión, y vuelvo a la Conferencia y Festival Internacional de Relaciones Públicas. En nuestro manifesto de la Conferencia establecemos que las Relaciones Públicas, cuando se practican profesionalmente, son una fuerza para el bien. Y están a la búsqueda de habilidades, diversidad, acciones éticas y profesionales y fundamentalmente establece que "las Relaciones Públicas bien implementadas son absolutamente interactivas".

La práctica adecuada de las Relaciones Públicas sólo es posible en una democracia donde existe la comunicación de doble vía. Justamente en este mes hemos visto cómo la función de las Relaciones Públicas quedó trastocada en los Juegos Olímpicos. El recorrido de la antorcha olímpica no hizo más que resaltar la imagen del Estado Chino generando incluso que hasta los "guardaespaldas" brillaran más que el emblemático símbolo olímpico.

La definición de Relaciones Públicas está impactada por las diferencias culturales y ciertas consideraciones, pero, en el Reino Unido y de acuerdo a CIPR y otros miembros del Global Alliance, Relaciones Públicas no es el "arte oscuro", de "manipulación" o de "envolvimiento". Todo lo contrario. Las Relaciones Públicas se ocupan de construir relaciones duraderas con diferentes grupos a través de una comunicación efectiva de "doble vía". En el ámbito de los negocios se trata de ayudar a la organización a alcanzar sus objetivos, pero sus funciones van mucho más allá. Ligadas a la libertad de expresión, las Relaciones Públicas pueden proporcionar una voz a aquellos que pueden no tener una necesariamente, y este es el motivo por el cual las Relaciones Públicas no pueden existir más que en una democracia.

La Conferencia y Festival Internacional de Relaciones Públicas fue realmente un evento global; la Conferencia reunió más de 200 profesionales de todo el mundo para compartir ideas y experiencias, y reconocer los trabajos destacados de Relaciones Públicas implementados a nivel mundial. Delega- 
dos representando más de 34 países asistieron a los dos días de conferencia. Con más de 20 sesiones con variadas temáticas y casos de estudio -como por ejemplo, el uso de las Relaciones Públicas para desafiar la marginalidad de personas discapacitadas y minorías étnicas, entre muchas otras- la Conferencia no pudo estar más lejos del estereotipo de las Relaciones Públicas promovido por los medios masivos.

Mirando en mi bola de cristal los próximos 60 años, veo a CIPR continuando con el camino que ha iniciado y mantenido. Nos veo continuando el liderazgo de la industria de las Relaciones Públicas en el Reino Unido y elevando los estándares profesionales, para ser aún más relevantes para los miembros individuales, e incrementar la cantidad de nuevos miembros; y también nos veo comunicando en una voz mucho más alta el valor de las Relaciones Públicas al mundo entero, construyendo relaciones y trabajando más estrechamente con otras organizaciones nacionales de Relaciones Públicas a través de la Global Alliance. Y nos veo trabajando en forma mucho más cercana con ustedes aquí en Argentina, compartiendo experiencias y desarrollándonos en forma conjunta.

Tenemos mucho que aprender unos de otros, ayudándonos a actuar a favor del bien público.

Summary: Next content translated to Spanish reproduces the contents, of the lecture delivered by Elisabeth Lewis Jones in the 'First International Conference of Public Relations, organized in occasion of the celebration of the $50^{\circ}$ anniversary of the Professional Council of Public Relations of Argentina, in Buenos Aires, the 26th 2008, with the participation of international referring professionals of the industry. Lewis Jones, in his function of President of the Chartered Institute of Public Relations (CIPR/Institute of Public Relations of Great Britain) reflects about the global and international scene of the public relations, emphasizing their contribution to the social development through the management of an effective communication.

Keywords: Chartered Institute of Public Relations - Professional Council of Public Relations of Argentina- Public Relations - Corporate Reputation - Investors Relations - Internal Communication - Marketing Communications - corporate image - organization - communicational management - plans of communication - public - corporate communication - institutional communication

Resumo: A seguir se reproduz o conteúdo traduzido ao espanhol, da exposição feita por Elisabeth Lewis Jones na 'Primeira Conferência Internacional de Relações Públicas', organizada em ocasião da celebração do $50^{\circ}$ aniversário pelo Conselho Profissional de Relações Públicas da República Argentina, em Buenos Aires, o 26 de agosto de 2008, com a participaçao de referentes internacionais da indústria. Lewis Jones, na sua função de Presidenta do Chartered Institute of Public Relations (CIPR / Instituto de Relaçoes Públicas de Gra-Bretanha) reflexiona a respeito do palco global e internacional das relações públicas, destacando o aporte que estas realizam ao desenvolvimento social através da gestão de uma comunicação efetiva.

Palavras chave: Chartered Institute of Public Relations - Conselho Profissional de Relações Públicas da República Argentina - Relações Públicas - Reputação Corporativa - Relações com os Investidores - Comunicação Interna - Comunicação de Marketing - imagem corporativa - organização - gestão comunicacional - planos de comunicação - públicos - comunicação corporativa - comunicação empresária - comunicação institucional 